TRANSACTIONS OF THE

AMERICAN MATHEMATICAL SOCIETY

Volume 350, Number 6, June 1998, Pages 2457-2471

S 0002-9947(98)01941-2

\title{
FIXED POINT SETS OF DEFORMATIONS OF POLYHEDRA WITH LOCAL CUT POINTS
}

\author{
PETER WOLFENDEN
}

\begin{abstract}
A locally finite simplicial complex $X$ is said to be 2-dimensionally connected if $X-\{$ local cut points of $X\}$ is connected. Such spaces exhibit "classical" behavior in that they all admit deformations with one fixed point, and they admit fixed point free deformations if and only if the Euler characteristic is zero. A result of G.-H. Shi implies that, for non 2-dimensionally connected spaces, the fixed point sets of deformations are equivalent to the fixed point sets of certain combinatorial maps which he calls good displacements. U. K. Scholz combined Shi's results with a theorem of P. Hall to obtain a characterization of all finite simplicial complexes which admit fixed point free deformations. In this paper we begin by explicitly capturing the combinatorial structure of a non 2-dimensionally connected polyhedron in a bipartite graph. We then apply an extended version of Hall's theorem to this graph to get a realization theorem which gives necessary and sufficient conditions for the existence of a deformation with a prescribed finite fixed point set. Scholz's result, and a characterization of all finite simplicial complexes without fixed point free deformations that admit deformations with a single fixed point follow immediately from this realization theorem.
\end{abstract}

\section{INTRODUCTION}

Throughout this paper, $X$ will be a connected polyhedron (or, equivalently, a locally finite connected simplical complex). The Euler characteristic of $X$ is denoted $\chi(X)$, the boundary of $X$ is denoted $\partial X$, and we adopt the notation $f r_{X}(J)$ to denote the frontier in $X$ of a subpolyhedron $J$, which is defined to be the space (closure of $J$ in $X)$ - $\operatorname{int}(J)$. The cardinality of a finite set $F$ is denoted $|F|$, the fixed point set of a map $f: X \rightarrow X$ is denoted $f i x(f)$, and the minimum number of fixed points in the homotopy class of $f$ is $M F(f)=\min \{|f i x(g)|: g$ homotopic to $f\}$. A deformation of $X$ is a map homotopic to $i d_{X}$, the identity map.

A vertex $v$ of $X$ is a local cut point of $X$ if there exists a connected neighborhood $N$ of $v$ in $X$ such that $N-v$ is disconnected.

The polyhedron $X$ is 2-dimensionally connected if the space

$$
X-\{\text { local cut points of } X\}
$$

is connected. This definition is equivalent to the much longer one appearing in [5], though it makes no mention of 2-simplexes. A connected space with no local cut points is clearly 2-dimensionally connected.

Received by the editors June 20, 1995 and, in revised form, August 12, 1996.

1991 Mathematics Subject Classification. Primary 54C99; Secondary 05C90.

Key words and phrases. Deformation, fixed point, local cut point, part, welding vertex, marriage theorem. 
The following classical result (see [2, Thm. 2.4, p. 91]) shows that 2-dimensionally connected spaces are well-behaved with regard to fixed points of deformations:

Theorem 1.1. Given a compact 2-dimensionally connected polyhedron $X$,

$$
M F\left(i d_{X}\right)= \begin{cases}0 & \text { for } \chi(X)=0 \\ 1 & \text { for } \chi(X) \neq 0 .\end{cases}
$$

The study of polyhedra which are not 2-dimensionally connected began with [5], which established an equivalence between fixed point sets of deformations and fixed point sets of "good displacements" (for a definition see the next section). This equivalence was then combined with a combinatorial result [1] to give a characterization [4] of all compact polyhedra for which $M F\left(i d_{X}\right)=0$. The main result of this paper is a realization theorem, based on an extension of Hall's theorem, which gives necessary and sufficient conditions for the existence of a deformation with a prescribed finite fixed point set. The characterization of compact polyhedra $X$ for which $M F\left(i d_{X}\right)=1$ follows immediately from this realization theorem, and the $M F\left(i d_{X}\right)=0$ characterization is easily recovered as well. This completes the taxonomy of compact, non 2-dimensionally connected polyhedra which exhibit "classical fixed point behavior" as in Theorem 1.1. All other polyhedra have $M F\left(i d_{X}\right)>1$. Note that the results of [5] hold for polyhedra which are not necessarily compact, but that the fixed point characterizations which appear in this paper are stated only for compact polyhedra. This is because the combinatorial results used in this paper rely on an induction which requires a certain graph representation of the polyhedron in question to be finite, and noncompact polyhedra may have infinite graph representations.

\section{The Theorems of Shi And Scholz}

Most of the definitions which follow come from [5]. The few changes I have made are intended only to streamline the presentation, and in adding new terminology I have attempted to preserve the industrial flavor of the nomenclature.

Let the local cut points of a polyhedron $X$ be called welding vertices of $X$ and denote them $v_{i}, i=1,2, \ldots$. The set of all welding vertices is called the welding set of $X$ and is denoted $W S(X)$. A weld $W$ of $X$ is a subset of $W S(X)$, and we let $W(X)$ denote the set of welds of $X$ (that is, $W(X)$ is the power set of $W S(X)$ ).

A part $P$ of $X$ is the closure (in $X$ ) of a component of $X-W S(X)$. Note that $f r_{X}(P)$ consists entirely of welding vertices of $X$, but that the welding vertices of $X$ which belong to $P$ need not all lie in $f r_{X}(P)$. Figure 1 depicts a connected 2-dimensional polyhedron with 5 parts and 5 welding vertices, henceforth referred to as Example 1. In Example 1, parts $P_{1}, P_{2}$, and $P_{3}$ are line segments, $P_{4}$ is homotopy equivalent to a circle, and $P_{5}$ is a 2 -sphere.

Let $P(X)=\left\{P_{1}, P_{2}, \ldots\right\}$ be the set of parts of $X$. An assembly $A$ in $X$ is a subpolyhedron of $X$ that is a union of parts of $X$. Let $W S(A)=W S(X) \cap A$ be the set of welding vertices of $X$ which belong to $A$, and let $W(A)=W(X) \cap A$ be the set of welds of $A$ (that is, $W(A)$ is the power set of $W S(A)$ ). In Example 1, taking, say $A=P_{4} \cup P_{5}$ gives $W S(A)=\left\{v_{3}, v_{4}, v_{5}\right\}$. Define the $P$-neighborhood $N_{P}(T)$ of a set of welding vertices $T \subseteq W S(X)$ to be the assembly consisting of parts which meet $T$. For instance, in Example 1, $N_{P}\left(\left\{v_{1}, v_{2}\right\}\right)=P_{1} \cup P_{2} \cup P_{3}$. Finally, let $A(X)$ denote the set of all assemblies in $X$. 


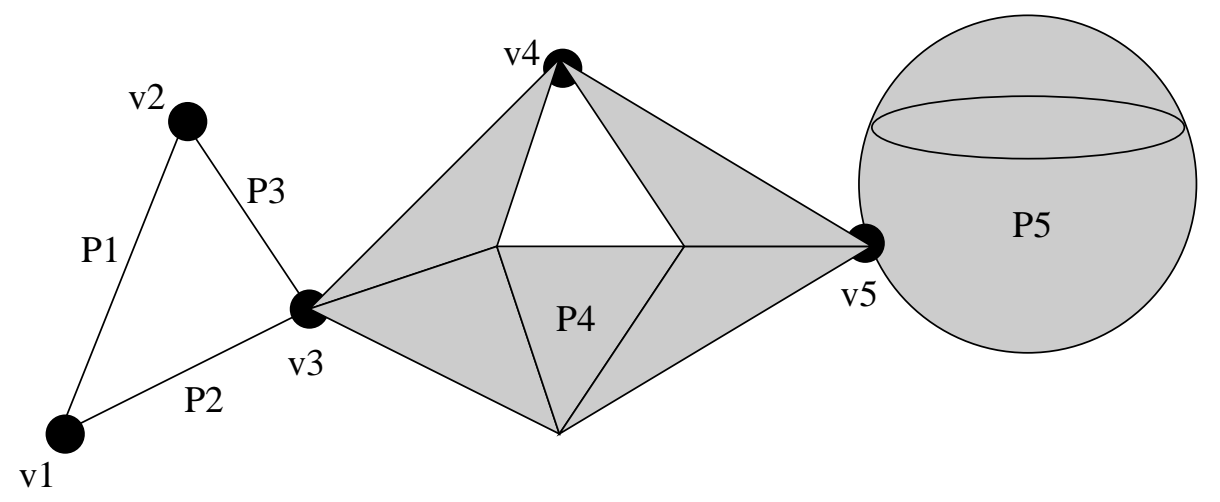

FIGURE 1

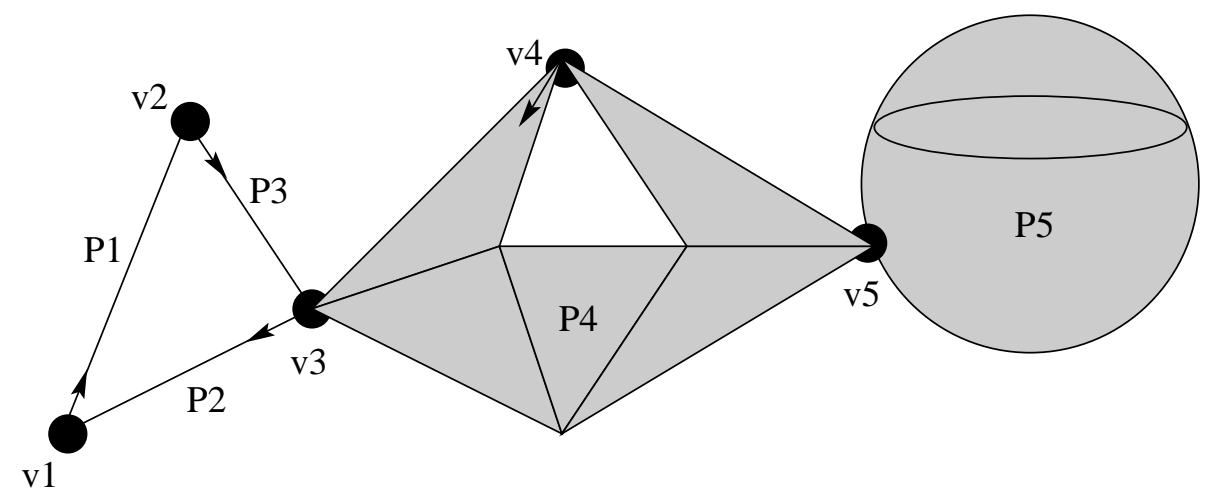

FiguRE 2

A good displacement (GD) of $X$ is a function

$$
g: W S(X) \rightarrow P(X) \cup W S(X)
$$

with the following properties:

(i) For all $v \in W S(X), v \in g(v)$. This means $g(v) \in P(X)$ unless $v=g(v)$, in which case we say that $v$ is a fixed point of $g$.

(ii) If $g(v) \neq v$ for all $v \in f r_{X}(P)$ for some $P$ (that is, $g$ has no fixed points in $P)$, then $\left|\left\{v \in f r_{X}(P): g(v) \notin P\right\}\right|=\chi(P)$.

Denote the set of all GDs of $X$ by $G D(X)$ and, for $g \in G D(X)$, define

$$
f i x(g)=\{v \in W S(X): v=g(v)\} .
$$

A GD is effectively a set of "directions" in which to move the welding vertices of $X$. A GD $g_{1}$ on Example 1 sending $v_{1}$ to $P_{1}, v_{2}$ to $P_{3}, v_{3}$ to $P_{2}, v_{4}$ to $P_{4}$, and fixing $v_{5}$ is shown in Figure 2. Any deformation of $X$ has a naturally associated GD, and any given GD may be extended to a deformation of $X$. This relationship between GDs and deformations of $X$ is made explicit in the following pair of results, which are a reformulation of Lemmas 4 and 5 on pp. 382-385 of [5] (see also [2, pp. 91-106]):

Lemma 2.1. For $X$ a locally finite complex, and $g$ a $G D$ of $X$, there exists a deformation $f$ of $X$ such that 
(i) $\left.f\right|_{W S(X)}=g$,

(ii) $f i x(f) \subseteq W S(X)$.

Lemma 2.2. For $X$ a locally finite complex, and $f$ a deformation of $X$, there exists a GD $g$ of $X$ such that $|f i x(g)| \leq|f i x(f)|$. Further, if fix $(f) \subseteq W S(X)$, then $f i x(g)=f i x(f)$.

The following theorem is an obvious consequence of the above lemmas:

Theorem 2.3 ([5]). For $X$ a locally finite complex,

$$
M F\left(i d_{X}\right)=\min \{|f i x(g)|: g \in G D(X)\} .
$$

The significance of this theorem is that it reduces the problem of finding the minimum number of fixed points among all deformations to the finite combinatorial problem of finding a GD with the smallest possible number of fixed points. In principle, we could calculate this smallest number by checking all possible GDs; and, for polyhedra with few parts and welding vertices, it is easy to program a computer to do so.

A slightly stronger version of Theorem 2.3 comes as another direct consequence of Lemmas 2.1 and 2.2, and will be used later in the proof of the Characterization Theorem 5.2:

Theorem 2.4. For $X$ a locally finite complex, a set $F \subseteq W S(X)$ is the fixed-point set of some deformation $f$ of $X$ if and only if it is the fixed point set of some GD $g$ of $X$.

To produce characterizations of spaces which admit deformations with certain fixed point sets we need some tools from graph theory. These are presented in Sections 3 and 4 and, as a consequence of the development, we will obtain a simple proof of the following:

Theorem 2.5 ([4]). A compact, connected polyhedron $X$ which is not 2-dimensionally connected admits a fixed point free deformation if and only if both the following conditions hold:

(I) $\chi(A) \geq 0$ for all $A \in A(X)$,

(II) $\chi(X)=0$.

Observe that Example 1 satisfies condition (II) of Theorem 2.5, but not condition (I). By considering the possible GDs of this polyhedron, the reader is invited to check that minimum number of fixed points of deformations for this space is 1 .

\section{Polyhedra, Graphs and Matchings}

Observe that the Euler characteristic of an assembly may be computed in terms of its constituent parts as follows:

$$
\chi(A)=\left(\sum_{P \subset A}\left(\chi(P)-\left|f r_{X}(P)\right|\right)\right)+|W S(A)| .
$$

Define the attraction of a part $P$ to be $\operatorname{att}(P)=\left|f r_{X}(P)\right|-\chi(P)$. For instance, in Example 1 we have $\operatorname{att}\left(P_{4}\right)=2$ and $\operatorname{att}\left(P_{5}\right)=-1$. This definition has two convenient properties:

First, we may rewrite the Euler characteristic formula as:

$$
\chi(A)=|W S(A)|-\sum_{P \subset A} \operatorname{att}(P),
$$


which, if we define the attraction of an assembly by

$$
\operatorname{att}(A)=\sum_{P \subset A} \operatorname{att}(P)
$$

may be written in the more compact form

$$
\chi(A)=|W S(A)|-\operatorname{att}(A) .
$$

Second, observe that if $\chi(P) \geq 0$ and $\left|f r_{X}(P)\right| \geq \chi(P)$ then, by the definition of a GD, $\operatorname{att}(P)$ is the number of welding vertices that must be sent into $P$ by any GD $g$ of $X$ with $f i x(g) \cap f r_{X}(P)=\emptyset$. If $\chi(P)<0$ or $\left|f r_{X}(P)\right|<\chi(P)$, then any GD of $X$ must, by definition, have a fixed point in $f r_{X}(P)$.

All the information we need in order to compute $M F\left(i d_{X}\right)$ is contained in the labeled bipartite graph constructed from $X$ as follows:

$$
\mathcal{G}(X)=(P(X), W S(X), \mathcal{E}=\{(P, v): P \in P(X), v \in W S(X) \cap P\}) .
$$

In other words, $P(X)$ and $W S(X)$ are the two vertex sets of $\mathcal{G}(X)$, and the edge set $\mathcal{E}$ connects each part in $P(X)$ to all the welding vertices in its frontier. Figure 3 shows the graph constructed from Example 1. For an assembly $A \subseteq A(X)$, define:

$$
\Gamma(A)=\{v \in W S(X):(P, v) \in \mathcal{E} \text { for some } P \subseteq A\} .
$$

For a weld $T \subseteq W(X)$, define

$$
\gamma(T)=\{P \in P(X) \mid(P, v) \in \mathcal{E} \text { for some } v \in T\} .
$$

Observe that any GD $g$ of $X$ may be represented by a subset $M_{g}$ of $\mathcal{E}$ defined as follows:

$$
M_{g}=\{(g(v), v) \in \mathcal{E}: v \neq g(v)\} .
$$

In other words, each vertex movement prescribed by $g$ has an associated edge in $M_{g}$. Notice that the definition does not permit any edges in $M_{g}$ to share a vertex in $W S(X)$. The heavy lines in Figure 4 represent $M_{g_{1}}$ for the GD $g_{1}$ on Example 1 defined in the last section. Conversely, we have conditions on an edge set $M$ which guarantee an associated GD:

Observation 3.2. If $M \subseteq \mathcal{E}$, then $M=M_{g}$ for some $g \in G D(X)$ if and only if both these condition hold:

(i) No two edges in $M$ share a vertex in $W S(X)$ and

(ii) for each $P \in P(X)$, either

(a) $\chi(P) \geq 0$, att $(P) \geq 0$, and $|\{(P, v) \in M\}|=\operatorname{att}(P)$, or

(b) fix $(g) \cap \Gamma(P) \neq \emptyset$ (in other words there is a fixed point of $g$ in $\left.f r_{X}(P)\right)$.

Considering the special case in which $g$ has no fixed points, we have the following:

Remark 3.3. $M=M_{g}$ for some fixed point free GD $g$ if and only if all three of the following conditions hold:

(i) No two edges in $M$ share a vertex in $W S(X)$.

(ii) For each $P \in P(X)$,

$$
\chi(P) \geq 0, \operatorname{att}(P) \geq 0 \text {, and }|\{(P, v) \in M\}|=\operatorname{att}(P) .
$$

(iii) Every $v \in W S(X)$ is the endpoint of some edge $e \in M$. 


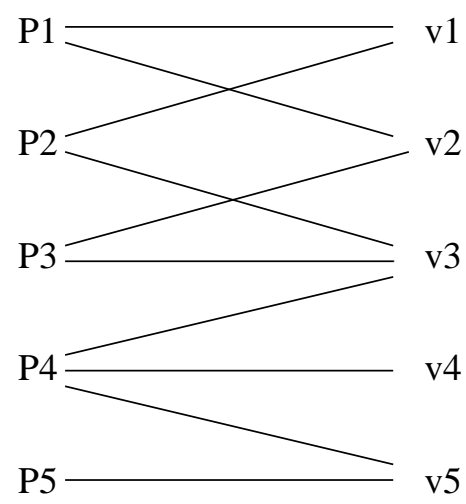

FiguRE 3

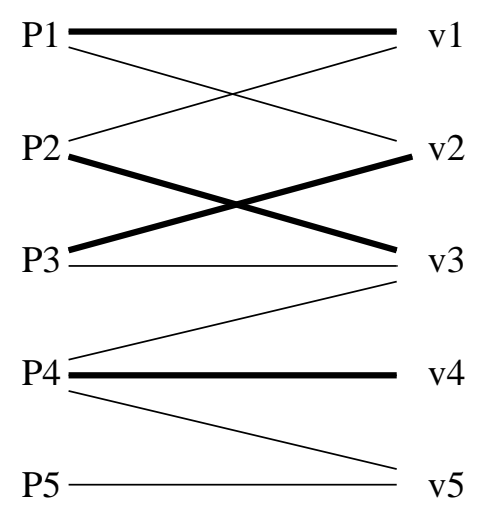

Figure 4

Also, considering the special case in which $g$ has a prescribed fixed point set $F$, we have the following:

Remark 3.4. $M=M_{g}$ for some GD $g$ with fixed point set $F$ if and only if all four of the following conditions hold:

(i) No two edges in $M$ share a vertex in $W S(X)$.

(ii) For each $P \in P(X)-\gamma(F)$,

$$
\chi(P) \geq 0, \operatorname{att}(P) \geq 0 \text {, and }|\{(P, v) \in M\}|=\operatorname{att}(P) .
$$

(iii) Every $v \in W S(X)-F$ is the endpoint of some edge $e \in M$.

(iv) No edge in $M$ meets $F$.

\section{Hall's Marriage Theorem}

This section is devoted to stating Hall's marriage theorem [1] in graph theoretic terms, and to a matching theorem [3] which gives a natural extension of Hall's theorem. Hall's theorem was originally stated in terms of representatives of subsets, but may be interpreted as giving the conditions under which a perfect matching (see below) exists in a bipartite graph. The name "marriage theorem" derives from 
a scenario in which the bipartite graph under consideration represents marital compatibilities between unwed adults in an isolated heterosexual population, and the task of finding a perfect matching corresponds to the task of arranging monogamous marriages between compatible partners so that everyone has a spouse.

For a general bipartite graph $\mathcal{G}=(\mathcal{A}, B, E)$, we define:

$$
\begin{gathered}
\Gamma(S)=\{b \in \mathcal{B}:(a, b) \in \mathcal{E} \text { for some } a \in S\} \text { for } S \subseteq \mathcal{A}, \text { and } \\
\gamma(T)=\{a \in \mathcal{A}:(a, b) \in \mathcal{E} \text { for some } b \in T\} \text { for } T \subseteq \mathcal{B} .
\end{gathered}
$$

In what follows, we denote strict inclusion by $\subset$, whereas $\subseteq$ allows equality between sets.

A matching in a bipartite graph $\mathcal{G}$ is a subset $\mathcal{M}$ of $\mathcal{E}$ such that no two elements of $\mathcal{M}$ share a vertex. A matching $\mathcal{M}$ saturates a vertex set $S$ in $\mathcal{G}$ if every vertex $v \in S$ is the endpoint of an edge $e \in \mathcal{M}$. A matching is said to be perfect if it saturates both $\mathcal{A}$ and $\mathcal{B}$.

The above definitions are standard. We now make three more, which will be convenient for stating and proving the results in this section:

For a vertex subset $U$ of $\mathcal{G}$, we define a $U$-semimatching in $\mathcal{G}$ to be a subset $\mathcal{M}$ of $\mathcal{E}$ such that no two edges of $\mathcal{M}$ share an endpoint in $U$ (though they may share endpoints outside $U$ ).

For $S \subseteq \mathcal{A}$, condition $C_{S}(\mathcal{G})$ holds if $\left|S^{\prime}\right| \leq\left|\Gamma\left(S^{\prime}\right)\right|$ for all $S^{\prime} \subseteq S$.

For $T \subseteq \mathcal{B}$, condition $C_{T}(\mathcal{G})$ holds if $\left|T^{\prime}\right| \leq\left|\gamma\left(T^{\prime}\right)\right|$ for all $T^{\prime} \subseteq T$.

Note that $C_{S}(\mathcal{G})$ implies $|S| \leq|\mathcal{B}|$, and $C_{T}(\mathcal{G})$ implies $|T| \leq|\mathcal{A}|$.

When there is more than one graph in the discussion, we will write $\mathcal{A}(\mathcal{G}), \mathcal{B}(\mathcal{G})$, and $\mathcal{E}(\mathcal{G})$ to denote the vertex and edge sets associated to $\mathcal{G}$. We will use $\Gamma_{\mathcal{G}}$ and $\gamma_{\mathcal{G}}$ to denote the set functions associated to $\mathcal{G}$, and we will write $\mathcal{G}(X)$ to indicate that the graph was constructed from the space $X$ as in Section 3.

We now have all the notation we need in order to present the results of this section. Proofs of these well-known results follow easily from the material in many texts on matching theory, but [3] in particular has a complete proof of Theorem 4.5 .

Theorem 4.1 (Hall's Marriage Theorem [1]). A bipartite graph $\mathcal{G}$ admits a matching which saturates $\mathcal{A}$ if and only if $C_{\mathcal{A}}(\mathcal{G})$ is satisfied.

If we translate Theorem 4.1 into the marriage-arrangement scenario by letting the vertices in $\mathcal{A}$ represent men and the vertices in $\mathcal{B}$ represent women, then the theorem guarantees that we can monogamously marry off all the men if and only if every collection of men is compatible with a large enough collection of women.

Corollary 4.2. Let $\mathcal{G}$ be a bipartite graph with labeling $l: \mathcal{A}(\mathcal{G}) \rightarrow \mathbb{Z}_{+}$. There exists a $\mathcal{B}$-semimatching $\mathcal{M}$ in $\mathcal{G}$ such that $|\{(a, b) \in \mathcal{M}\}|=l(a)$ for all $a \in \mathcal{A}(\mathcal{G})$ if and only if for all $S \subseteq \mathcal{A}(\mathcal{G})$,

$$
\sum_{a \in S} l(a) \leq\left|\Gamma_{\mathcal{G}}(S)\right|
$$

Corollary 4.3. A bipartite graph $\mathcal{G}$ admits a matching which saturates a vertex subset $S \subseteq \mathcal{A}$ if and only if $C_{S}(\mathcal{G})$ is satisfied.

Corollary 4.4. A bipartite graph $\mathcal{G}$ admits a matching which saturates a vertex subset $T \subseteq \mathcal{B}$ if and only if $C_{T}(\mathcal{G})$ is satisfied. 
Theorem 4.5 ([3, pp. 113-114]). Let $\mathcal{G}$ be a bipartite graph, and let $S \subseteq \mathcal{A}$ and $T \subseteq \mathcal{B}$ be vertex subsets. Let $\mathcal{M}$ be a matching in $\mathcal{G}$ saturating $S$, and let $\mathcal{M}^{\prime}$ be a matching in $\mathcal{G}$ saturating $T$. Then from the edges in $\mathcal{M}$ and $\mathcal{M}^{\prime}$ one can construct a matching in $\mathcal{G}$ saturating both $S$ and $T$.

Theorem 4.6. Let $\mathcal{G}$ be a bipartite graph, and let $S \subseteq \mathcal{A}$ and $T \subseteq \mathcal{B}$ be vertex subsets. There exists a matching in $\mathcal{G}$ saturating both $S$ and $T$ if and only if both $C_{S}(\mathcal{G})$ and $C_{T}(\mathcal{G})$ hold.

Corollary 4.7. Let $\mathcal{G}$ be a bipartite graph with labeling $l: \mathcal{A} \rightarrow \mathbb{Z}_{+}$and vertex subset $S \subseteq \mathcal{A}$. There exists a $\mathcal{B}$-semimatching $\mathcal{M}$ in $\mathcal{G}$ saturating both $S$ and $\mathcal{B}$ such that $|\{(a, b) \in \mathcal{M}\}|=l(a)$ for all $a \in S$ if and only if both of the following conditions hold: for all $S^{\prime} \subseteq S$,

$$
\sum_{a \in S^{\prime}} l(a) \leq\left|\Gamma\left(S^{\prime}\right)\right|
$$

for all $T \subseteq \mathcal{B}$,

$$
\sum_{a \in \gamma(T)} l(a) \geq|T|
$$

Note that condition $(* *)$ is dual to condition $(*)$ in Corollary 4.2, and condition $(\dagger)$ is a restriction of condition $(*)$ to the subset $S$ of $\mathcal{A}$.

\section{Fixed Point Characterizations}

We begin by considering fixed-point free deformations. By Theorem 2.4, a polyhedron $X$ admits a fixed point free deformation if and only if there exists a fixed point free GD of $X$. Now recall Remark 3.3, which says that a subset $\mathcal{M}$ of $\mathcal{E}(\mathcal{G}(X))$ represents some fixed point free GD of $X$ if and only if all three of the following conditions hold:

(i) No two edges in $\mathcal{M}$ share a vertex in $W S(X)$.

(ii) For each $P \in P(X)$,

$$
\chi(P) \geq 0, \operatorname{att}(P) \geq 0, \text { and }|\{(P, v) \in \mathcal{M}\}|=\operatorname{att}(P) .
$$

(iii) Every $v \in W S(X)$ is the endpoint of some edge in $\mathcal{M}$.

Throughout the rest of this section we will require that our polyhedron $X$ be compact, so as to guarantee that $\mathcal{G}(X)$ is a finite graph. We define a labeling on $\mathcal{A}(\mathcal{G}(X))$ by carrying over the attraction defined on $P(X)$ in the natural way. The "attraction" of each vertex $a \in \mathcal{A}(\mathcal{G}(X))$ is defined to be the attraction of the corresponding part $P \in P(X)$.

Recall that if $\chi(P)<0$ or att $(P)<0$ for some part $P$ of $X$, then by definition all GDs of $X$ must have at least one fixed point. Condition (ii) implies that, for $\mathcal{G}=\mathcal{G}(X)$, the attraction labeling on $\mathcal{A}(\mathcal{G})$ makes $\mathcal{G}$ into a graph satisfying the hypotheses of Corollary 4.2 , and that $\mathcal{M}$ satisfies the condition

$$
|\{(P, v) \in \mathcal{M}\}|=\operatorname{att}(P)
$$

in the conclusion of the same result. Therefore Corollary 4.2 implies that $(*)$ is a necessary condition for the existence of a fixed point free GD on $X$, that is, for each $S \subseteq \mathcal{A}(\mathcal{G})$,

$$
\sum_{a \in S} \operatorname{att}(a) \leq|\Gamma(S)| .
$$


Taking $S=\mathcal{A}$ in the above inequality gives us $\sum_{a \in \mathcal{A}} a t t(a) \leq\left|\Gamma_{\mathcal{G}}(\mathcal{A})\right|$, where $\left|\Gamma_{\mathcal{G}}(\mathcal{A})\right| \leq|\mathcal{B}|=|W S(X)|$. By definition of our attraction labeling, we have $\sum_{P \in P(X)}$ att $(P) \leq|W S(X)|$. On the other hand, $\mathcal{M}$ can saturate $W S(X)$ only if $|W S(X)| \leq \sum_{P \in P(X)} \operatorname{att}(P)$, so the condition $|W S(X)|=\sum_{P \in P(X)} \operatorname{att}(P)$ is also necessary for the existence of a fixed point free GD on $X$.

So we have the following three necessary conditions for $X$ to admit a fixed point free GD:

(A) For all $P \in P(X), \chi(P) \geq 0$ and $\operatorname{att}(P) \geq 0$.

(B) For all $S \subseteq \mathcal{A}(\mathcal{G}(X)) \sum_{a \in S}$ att $(a) \leq\left|\Gamma_{\mathcal{G}}(S)\right|$.

(C) $|W S(X)|=\sum_{P \in P(X)} \operatorname{att}(P)$.

Corollary 4.2 implies that conditions (A) and (B) are sufficient to guarantee a $\mathcal{B}$-semimatching which satisfies condition (ii). If we add condition (C) then the semimatching also satisfies (iii), and so the conditions (A), (B), and (C) are sufficient to guarantee the existence of a fixed point free GD on $X$, and therefore a fixed point free deformation of $X$ by Lemma 2.4.

If we reformulate condition (B) using our formula (3.1) for the Euler characteristic of an assembly, we see that it becomes:

$$
\text { For all assemblies } A \text { in } A(X), \chi(A) \geq 0 \text {. }
$$

If we reformulate condition (C) using the same formula, it becomes $\chi(X)=0$.

We now show that (B) and (C) together imply (A). Clearly condition (B) implies that $\operatorname{att}(P) \leq\left|f r_{X}(P)\right|$, and hence $\chi(P) \geq 0$ for all $P \in P(X)$. So we need only to show that if (B) and (C) hold, then $\operatorname{att}(P) \geq 0$ for all $P \in P(X)$. For any $P \in P(X)$, consider the assembly $Y=(X-P) \cup f r_{X}(P) \in A(X)$. By the definition of Euler characteristic we have:

$$
\chi(X)=\chi(Y)+\chi(P)-\left|f r_{X}(P)\right| .
$$

Condition $(\mathrm{C})$ makes $\chi(X)=0$, so $\operatorname{att}(P)=\left|f r_{X}(P)\right|-\chi(P)=\chi(Y)$. Since (B) implies $\chi(Y) \geq 0$, we have $\operatorname{att}(P) \geq 0$ for all $P \in P(X)$. Thus we obtain the characterization from [4]:

Theorem $2.5([4])$. Given a compact polyhedron $X$, there exists a fixed-point-free deformation of $X$ if and only if both of the following conditions hold:

(I) For all assemblies $A$ of $X, \chi(A) \geq 0$.

(II) $\chi(X)=0$.

Suppose now that $F \subseteq W S(X)$ is a set of prescribed fixed points, and we wish to find a deformation of $X$ with fixed point set $F$. By Lemma 2.4 this is possible if and only if there exists a GD of $X$ with fixed point set $F$. Recall Remark 3.4, which says that a subset $\mathcal{M}$ of $\mathcal{E}(\mathcal{G}(X))$ represents such a GD if and only if all four of the following conditions hold:

(i) No two edges in $M$ share a vertex in $W S(X)$.

(ii) For each $P \in P(X)$ disjoint from $F$,

$$
\chi(P) \geq 0, \operatorname{att}(P) \geq 0 \text {, and }|\{(P, v) \in M\}|=\operatorname{att}(P) .
$$

(iii) Every $v \in W S(X)-F$ is the endpoint of some edge $e \in M$.

(iv) No edge in $M$ meets $F$.

If some $P \in P(X)$ is disjoint from $F$ and either $\chi(P)<0$ or att $(P)<0$, then no such GD can exist. But if each $P \in P(X)$ with either $\chi(P)<0$ or att $(P)<0$ contains a vertex of $F$, then the task of finding such a GD realizing the fixed 
point set $F$ is equivalent to that of finding a $\mathcal{B}$-semimatching $\mathcal{M}$ in $\mathcal{G}(X)$ with the following properties:

(i) for any $a \in \mathcal{A}$ disjoint from $F,|(a, b) \in \mathcal{M}|=\operatorname{att}(a)$,

(ii) $\mathcal{M}$ saturates $\mathcal{B}-F$,

(iii) $\mathcal{M}$ misses $F$ completely.

If we consider the subgraph of $\mathcal{G}$ defined as follows:

$$
\mathcal{G}^{\prime}=(\mathcal{A}, \mathcal{B}-F,\{(a, b) \in \mathcal{E}: b \notin F\})
$$

with vertex subset $S=\mathcal{A}-\gamma_{\mathcal{G}}(F) \subseteq \mathcal{A}\left(\mathcal{G}^{\prime}\right)$, then we see that finding a $\mathcal{B}$ semimatching $\mathcal{M}$ in $\mathcal{G}$ as described above is equivalent to finding a $\mathcal{B}$-semimatching $\mathcal{M}^{\prime}$ in $\mathcal{G}^{\prime}$ which saturates both $\mathcal{B}\left(\mathcal{G}^{\prime}\right)$ and $S$, and which has the property that $\left|(a, b) \in \mathcal{M}^{\prime}\right|=\operatorname{att}(a)$ for any $a \in \mathcal{A}\left(\mathcal{G}^{\prime}\right)$. Corollary 4.7 implies that such a $\mathcal{B}$ semimatching $\mathcal{M}^{\prime}$ exists in $\mathcal{G}^{\prime}$ if and only if both the following conditions hold:

$(\dagger)$ For all $S^{\prime} \subseteq S, \sum_{a \in S^{\prime}} a t t(a) \leq\left|\Gamma_{\mathcal{G}^{\prime}}\left(S^{\prime}\right)\right|$

$(* *)$ For all $T \subseteq \mathcal{B}-F, \sum_{a \in \gamma_{\mathcal{G}^{\prime}}(T)} \operatorname{att}(a) \geq|T|$.

Using formula (3.1) again for the Euler characteristic of an assembly, we may reformulate condition $(\dagger)$ as follows:

Each assembly $A$ in $X$ disjoint from $F$ has $\chi(A) \geq 0$.

Recalling that the $P$-neighborhood $N_{P}(W)$ of a weld $W$ in $X$ is the assembly composed of all the parts of $X$ which meet $W$, we may reformulate condition $(* *)$ as follows:

Each weld $W$ of $X$ disjoint from $F$ has $\operatorname{att}\left(N_{P}(W)\right) \geq|W|$.

So we have the following theorem:

Theorem 5.1 (Realization Theorem). There exists a deformation of a compact polyhedron $X$ with fixed point set $F \subseteq W S(X)$ if and only if all of the following conditions hold:

(I) Every $P \in P(X)$ with either $\chi(P)<0$ or att $(P)<0$ contains some vertex of $F$.

(II) Each assembly $A$ in $X$ disjoint from $F$ has $\chi(A) \geq 0$.

(III) Each weld $W$ of $X$ disjoint from $F$ has att $\left(N_{P}(W)\right) \geq|W|$.

Returning again to Example 1, if we take $F$ to be $v_{5}$ then conditions (I), (II), and (III) of the realization theorem are satisfied. If we take $F$ to be anything that does not contain $v_{5}$, then condition (I) fails.

Before considering some more examples, we remark that given any finite bipartite graph $\mathcal{G}=(\mathcal{A}, \mathcal{B}, \mathcal{E})$ with a non-negative labeling att $: \mathcal{A} \rightarrow \mathbb{Z}_{+}$, we may construct a finite 2-dimensional polyhedron $X_{\mathcal{G}}$ with $\mathcal{G}\left(X_{\mathcal{G}}\right)=\mathcal{G}$ as follows:

For each integer $n \geq 0$, define the finite polyhedron $Q_{n}$ to be a circle and $n$ 2-disks, each with its boundary identified with the circle by a degree 1 map. For each integer $n<0$, define the finite polyhedron $Q_{n}$ to be a 2-disk with $1-n$ disjoint open disks removed from the interior. Note that $\chi\left(Q_{n}\right)=n$ for all integers $n$. For each $a \in \mathcal{A}$, let $n_{a}=|\Gamma(a)|-\operatorname{att}(a)$, and let $P_{a} \simeq Q_{n_{a}}$. Let $P(\mathcal{G})$ be the disjoint union of all the $P_{a}$. For each $(a, b) \in \mathcal{E}$ choose a distinct point $v_{b}$ on the interior of $P_{a}$. Then refine the triangulation of each $P_{a}$ as needed to make the $v_{b}$ s into vertices of the polyhedron, and define $X_{\mathcal{G}}$ to be the identification space of $P(\mathcal{G})$ obtained by identifying $v_{b} \in P_{a}$ with $v_{b} \in P_{a^{\prime}}$ for all $(a, b),\left(a^{\prime}, b\right) \in \mathcal{E}$. Now observe that $|\Gamma(a)|=\left|f r_{X_{\mathcal{G}}}\left(P_{a}\right)\right|$, that $\operatorname{att}(a)=\operatorname{att}\left(P_{a}\right)$, and that $n_{a}=\chi\left(P_{a}\right)$. 
Since any two finite polyhedra with the same graph representation will have deformations which exhibit the same fixed point behavior, when describing examples it is enough to specify the graph $\mathcal{G}$ with labeling att and assume that the above procedure is used to generate a space $X_{\mathcal{G}}$ with the desired properties. In the figures which follow, a part with fewer than 3 welding vertices in its frontier is drawn as an edge with a label (the Euler characteristic). A part with three or more welding vertices would be drawn as a circle with the label inside. Welding vertices are represented by solid dots.

As an application of the realization theorem, we will now characterize all compact polyhedra $X$ with $M F\left(i d_{X}\right)=1$.

First, we define three conditions on $X$ :

(C1) For all $P \in P(X)$, we have $\chi(X) \geq 0$ and $\operatorname{att}(P) \geq 0$.

(C2) For all $A \in A(X)$, we have $\chi(A) \geq 0$.

(C3) For all $W \subseteq W S(X)$, we have $\operatorname{att}\left(N_{P}(W)\right) \geq|W|$.

Second, we define a subset of $W S(X)$ for each condition, which represents either the set of welding vertices shared by all assemblies for which the condition fails, or the whole welding set if the condition holds:

$$
\begin{gathered}
F_{1}=\left\{\begin{array}{l}
W S(X) \quad \text { if }(\mathrm{C} 1) \text { holds, } \\
\bigcap\left\{f r_{X}(P): P \in P(X) \text { with } \chi(P)<0 \text { or } \operatorname{att}(P)<0\right\} \quad \text { otherwise, }
\end{array}\right. \\
F_{2}=\left\{\begin{array}{l}
W S(X) \text { if }(\mathrm{C} 2) \text { holds, } \\
\bigcap\{W S(X) \cap A: A \in A(X) \text { with } \chi(A)<0\} \quad \text { otherwise, }
\end{array}\right. \\
F_{3}=\left\{\begin{array}{l}
W S(X) \text { if }(\mathrm{C} 3) \text { holds, } \\
\bigcap\left\{W: W \subseteq W S(X) \text { with } \operatorname{att}\left(N_{P}(W)\right)<|W|\right\} \quad \text { otherwise. }
\end{array}\right.
\end{gathered}
$$

Finally, let $\mathcal{F}=F_{1} \cap F_{2} \cap F_{3}$. If conditions (C1), (C2), and (C3) are all satisfied by $X$, then it is easy to check that the hypotheses of Theorem 2.5 are satisfied, and so $X$ admits a fixed point free GD. Suppose at least one of the three conditions fails for $X$. Then $X$ admits no fixed point free GD, but if $\mathcal{F} \neq \emptyset$, then it is easy to check that the hypotheses of Theorem 5.1, with $F$ any vertex of $\mathcal{F}$, are satisfied and so $X$ admits a GD with a single fixed point. If $\mathcal{F}=\emptyset$, then at least one of the hypotheses of Theorem 5.1 must still be violated when $F$ is taken to be any single vertex of $W S(X)$, so $M F\left(i d_{X}\right)>1$. Therefore we have:

Theorem 5.2 (Characterization Theorem). A compact polyhedron $X$ has $M F\left(i d_{X}\right)=1$ if and only if

(i) At least one of the following conditions fails for $X$ :

(C1) For all $P \in P(X)$, we have $\chi(X) \geq 0$ and att $(P) \geq 0$.

(C2) For all $A \in A(X)$, we have $\chi(A) \geq 0$.

(C3) For all $W \subseteq W S(X)$, we have $\operatorname{att}\left(N_{P}(W)\right) \geq|W|$.

and

(ii) The intersection of these three sets is nonempty:

$$
F_{1}=\left\{\begin{array}{l}
W S(X) \quad \text { if }(C 1) \text { holds, } \\
\bigcap\left\{f r_{X}(P): P \in P(X) \text { with } \chi(P)<0 \text { or att }(P)<0\right\} \text { otherwise. }
\end{array}\right.
$$




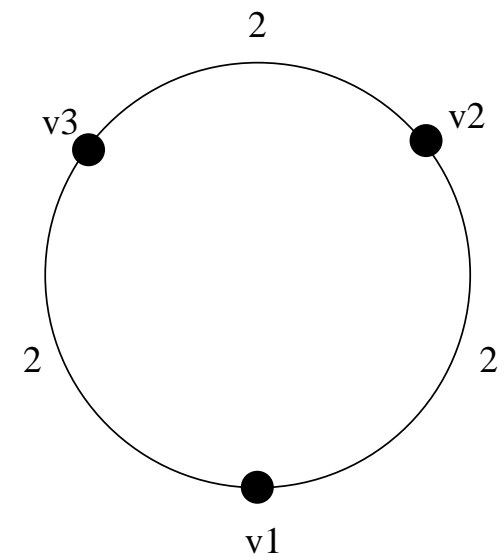

FiguRE 5

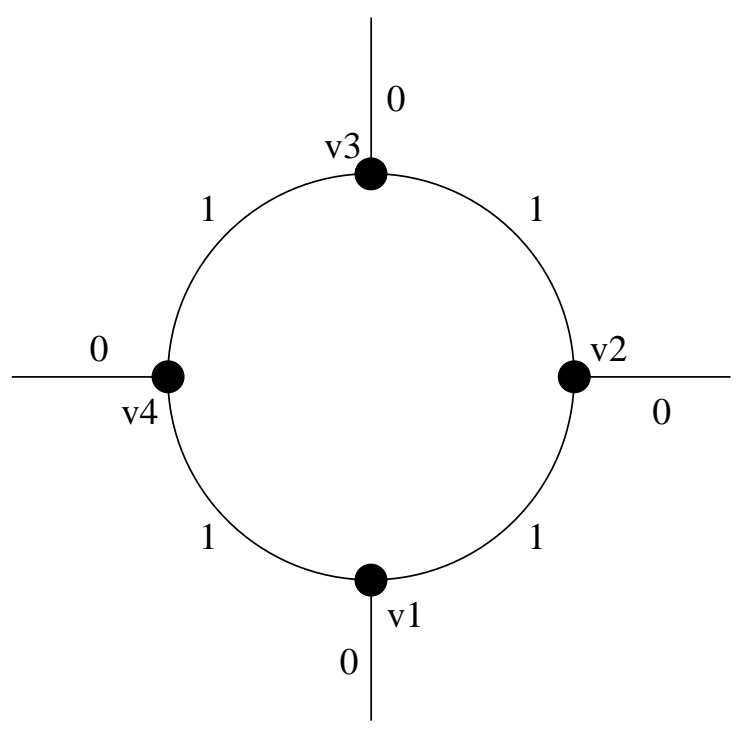

FigURE 6

$$
\begin{gathered}
F_{2}=\left\{\begin{array}{l}
W S(X) \text { if }(C 2) \text { holds, } \\
\bigcap\{W S(X) \cap A: A \in A(X) \text { with } \chi(A)<0\} \quad \text { otherwise, }
\end{array}\right. \\
F_{3}=\left\{\begin{array}{l}
W S(X) \text { if }(C 3) \text { holds, } \\
\bigcap\left\{W: W \subseteq W S(X) \text { with att }\left(N_{P}(W)\right)<|W|\right\} \quad \text { otherwise. }
\end{array}\right.
\end{gathered}
$$

Moreover, the set $\mathcal{F}$ is the set of possible single fixed points for a GD of $X$, hence by Theorem 2.4 of deformations $f$ of $X$ with $f i x(f) \subseteq W S(X)$.

In Example 1, condition (C1) fails with $F_{1}=\left\{v_{5}\right\}$, condition (C2) fails with $F_{2}=\left\{v_{1}, v_{2}, v_{3}, v_{4}, v_{5}\right\}$, and condition (C3) holds with $F_{3}=F_{2}$. So $\mathcal{F}=\left\{v_{5}\right\}$ as we observed earlier. 
We now consider two more examples:

Example 2, depicted in Figure 5 consists of three 2-spheres connected by three welding vertices.

In this example, conditions $(\mathrm{C} 1)$ and $(\mathrm{C} 2)$ hold, so $F_{1}=F_{2}=\left\{v_{1}, v_{2}, v_{3}\right\}$. Condition (C3) fails with $F_{3}=\left\{v_{1}, v_{2}, v_{3}\right\}$, so $\mathcal{F}=\left\{v_{1}, v_{2}, v_{3}\right\}$. Therefore given any welding vertex in Example 2, there is some deformation of Example 2 which fixes only that vertex.

Example 3 depicted in Figure 6, consists of four circles and four line segments connected by four welding vertices.

In this example, condition (C1) and (C3) hold, so $F_{1}=F_{3}=\left\{v_{1}, v_{2}, v_{3}, v_{4}\right\}$. Condition (C2) fails with $F_{2}=\emptyset$, so $\mathcal{F}=\emptyset$, and we conclude that all deformations of Example 3 must have at least two fixed points. The reader is invited to check that, in fact, the minimum number is two for Example 3.

\section{EXAMPLES}

In this section we build some families of compact polyhedra to demonstrate the connection between conditions $(\mathrm{C} 2)$ and $(\mathrm{C} 3)$ of the characterization theorem and the Euler characteristic.

Note that if condition (C2) holds for a compact polyhedron $X$, then $\chi(X) \geq 0$, since $X \in A(X)$. On the other hand, if condition condition (C3) holds for $X$, then $\chi(X) \leq 0$, because taking $W=W S(X)$ gives $N_{P}(W)=X$, and (C3) implies $\operatorname{att}(X)=|W S(X)|-\chi(X) \geq|W S(X)|$. So $\chi(X) \leq 0$.

Thus, if conditions (C1) and (C3) both hold for a compact polyhedron $X$, then either condition (C2) holds, in which case $\chi(X)=0$ and $M F\left(i d_{X}\right)=0$, or condition (C2) fails, in which case $\chi(X)<0$ and $M F\left(i d_{X}\right)>0$. We illustrate the latter situation by constructing an infinite family of compact polyhedra which satisfy conditions (C1) and (C3), but which realize all negative Euler characteristics and all minimum numbers as follows:

Let $X_{1}$ be a polyhedron with a single vertex and 2 edges attatched. This space is sometimes referred to as the "figure 8", and may be considered the wedge of two copies of $S^{1}$. Clearly $\chi\left(X_{1}\right)=-1$, and $M F\left(i d_{X_{1}}\right)=1$. We could now simply take the union of $n$ disjoint copies of $X_{1}$ to get a disconnected polyhedron with Euler characteristic $-n$ and minimum number $n$. But if to construct a connected example, let $X_{n}$ be the union of $n+1$ copies of $S^{1}$, arranged in a "chain" with $n$ welding vertices (see Figure 7 for a picture of $X_{4}$ ), then $X_{n}$ is a connected space with $\chi\left(X_{n}\right)=-n$. By considering the very restricted set of possible GDs of $X_{n}$, it is not difficult to show that at least every third vertex must be fixed, and that $M F\left(i d_{X_{n}}\right)=\lceil n / 3\rceil=$ the smallest integer not less than $n / 3$.

On the other hand, if conditions $(\mathrm{C} 1)$ and $(\mathrm{C} 2)$ hold for a compact polyhedron $X$, then either condition (C3) holds, in which case $\chi(X)=0$ and $M F\left(i d_{X}\right)=0$ by Theorem 2.5, or condition (C3) fails, in which case $\chi(X)>0$ and $M F\left(i d_{X}\right)>0$. We illustrate the latter situation by constructing another infinite family of compact

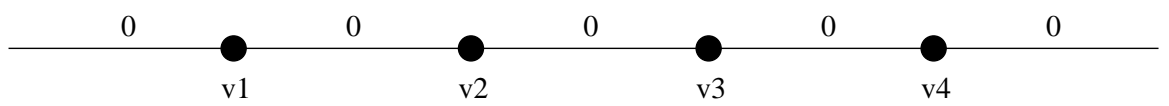

Figure 7 


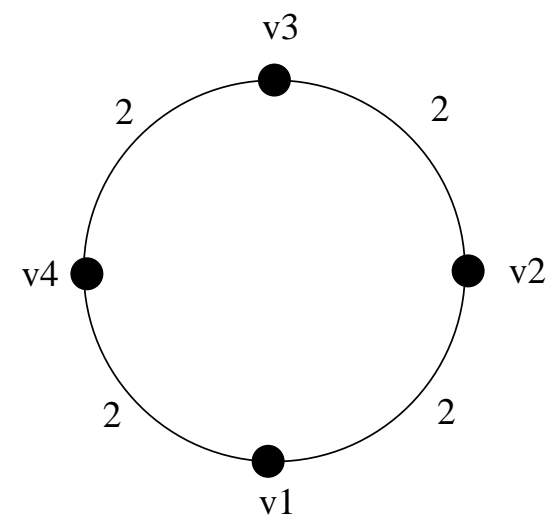

Figure 8

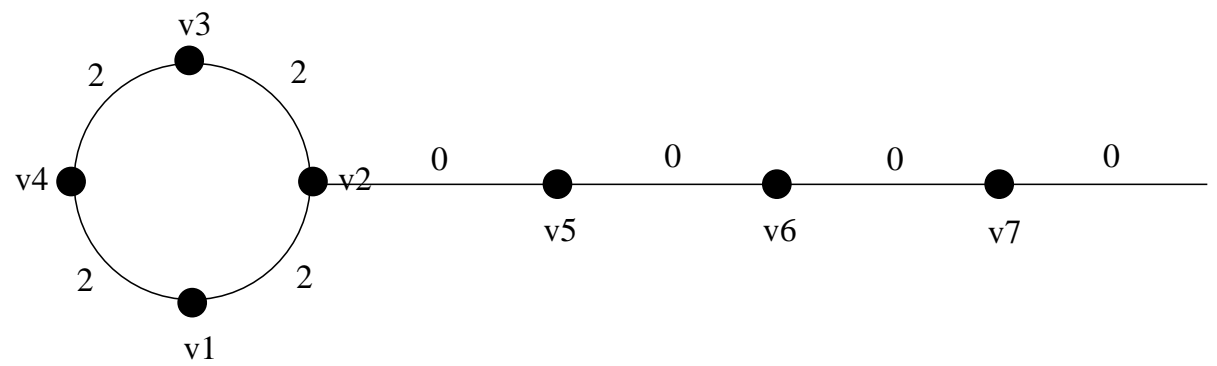

Figure 9

polyhedra which satisfy conditions $(\mathrm{C} 1)$ and $(\mathrm{C} 2)$, but which realize all positive Euler characteristics and all minimum numbers as follows:

Let $Y_{1}$ be a polyhedron with a single vertex, 2 edges, and 2 faces (2-simplices) attatched. Clearly $\chi\left(Y_{1}\right)=1$ and $M F\left(i d_{Y_{1}}\right)=1$. Let $Y_{n}$ be the union of $n$ copies of $S^{2}$, arranged in a "necklace" (see Figure 8 for a picture of $Y_{4}$ ), then $Y_{n}$ is a connected space with $\chi\left(Y_{n}\right)=n$. By considering the set of possible GDs of $Y_{n}$, it is not difficult to show that, again, at least every third vertex must be fixed, and that $M F\left(i d_{Y_{n}}\right)=\lceil n / 3\rceil$.

If condition (C1) fails, then it is difficult to say anything meaningful about $\chi(X)$ or $M F\left(i d_{X}\right)$, since each part of $X$ could have any Euler characteristic whatsoever, regardless of the number of welding vertices in its frontier. If condition (C1) holds, but both conditions $(\mathrm{C} 2)$ and $(\mathrm{C} 3)$ fail, then $X$ could have Euler characteristic zero but $M F\left(i d_{X}\right)>0$. We can combine the families of polyhedra described above to get a new family of examples which satisfy condition (C1) and have zero Euler characteristic, but which realize every positive minimum number as follows:

Let $Z_{n}$ be a copy of $Y_{n+1}$ joined at a vertex to a copy of $X_{n}$ (Figure 9 illustrates $\left.Z_{3}\right)$. Then $\chi\left(Z_{n}\right)=0$, and a slightly more subtle argument shows that $M F\left(i d_{Z_{n}}\right)$ increases with $n$. 


\section{REFERENCES}

1. P. Hall, On representatives of subsets, J. London Math Soc. 10 (1935), 26-30.

2. T.-H. Kiang, The Theory of Fixed Point Classes, Springer-Verlag, New York, 1989. MR 90h:55002

3. O. Ore, Theory of Graphs, Amer. Math Soc., Providence, RI, 1962. MR 27:740

4. U. K. Scholz, Fixed point free deformations on compact polyhedra, Nonlinear Functional Analysis and its Applications. D. Reidel Publishing Co., 1986, 387-392. MR 87k:55002

5. G.-H. Shi, On the least number of fixed points for infinite complexes, Pacific J. Math. 103, No. 2 (1982), 377-387. MR 85h:55005

AF BBn Planet, Inc., Palo Alto, California 94303

E-mail address: wolfen@bbnplanet.com 